\section{Percutaneous Transhepatic Cholangioscopic Lithotomy of a Common Bile Duct Stone through the Gallbladder Route}

\author{
Takako Maruyama, Akihiro Mori, Hideharu Tatebe, \\ Naoko Isono, Takayuki Asano, Noritsugu Ohashi, \\ Hiroshi Inoue, Shoudou Takegoshi \\ and Masataka Okuno
}

Key words: cholelithiasis, percutaneous transhepatic cholangioscopy, percutaneous transhepatic biliary drainage

(DOI: 10.2169/internalmedicine.46.7174)

Percutaneous transhepatic cholangioscopy (PTCS) is a valuable technique for the diagnosis and treatment of hepatobiliary lithiasis particularly when the transpapillary approach is difficult to accomplish (1). Moreover, percutaneous transhepatic gallbladder drainage (PTGBD) achieves the reduction in pyogenic inflammation of cholecystitis and cholangitis often accompanied by the lithiasis (2). PTCS performed after PTGBD is a well-tolerated method for the removal of gallbladder (GB) stones even in patients intolerable of surgery. However, when PTCS is introduced via the PTGBD route, difficulties are often encountered in the removal of stones in the common bile duct (CBD) that accompanies with GB stones $(3,4)$. Here, we report a patient with successful removal of a CBD stone as well as a number of stones in the GB by PTCS succeeding to PTGBD through the GB route.

A 77-year-old man visited us, complaining of upper abdominal colicky pain. Blood analyses showed increased levels of aspartate aminotransferase (562 IU/1), alkaline phosphatase (997 IU/l), $\gamma$-glutamyl transpeptidase (163 IU/l) and C-reactive protein $(1.6 \mathrm{mg} / \mathrm{dl})$. Ultrasonography and computed tomography showed a number of gallstones and signs of cholecystitis. Magnetic resonance cholangiopancreatography also demonstrated the presence of a stone in the CBD. Thus, we diagnosed the lesion as acute obstructive suppurative cholecystitis/cholangitis due to cholelithiasis. His unfavorable general conditions based on myelodysplastic syndrome suggested the preference for endoscopic removal of the stones over the surgical treatment. However, esophagogastroduodenoscope revealed difficulties in the transpapillary approach because he had a history of total gastrectomy due to gastric cancer and replacement operation for abdominal aortic aneurysm. Since the intrahepatic duct was not dilated on ultrasonography, we could not find any appropriate biliary duct for the transhepatic approach to CBD. Therefore, we performed PTGBD to eliminate biliary infection followed by PTCS aiming to remove the CBD and GB stones through the cholecystic route. A guide-wire was introduced into the CBD through the GB and cholecystic duct under fluoroscopy and then a PTGBD tube was inserted and reached the duodenum via the GB and CBD (Fig. 1A). Cholangiograhy using a PTGBD tube confirmed numerous stones in the gallbladder as well as a stone in the
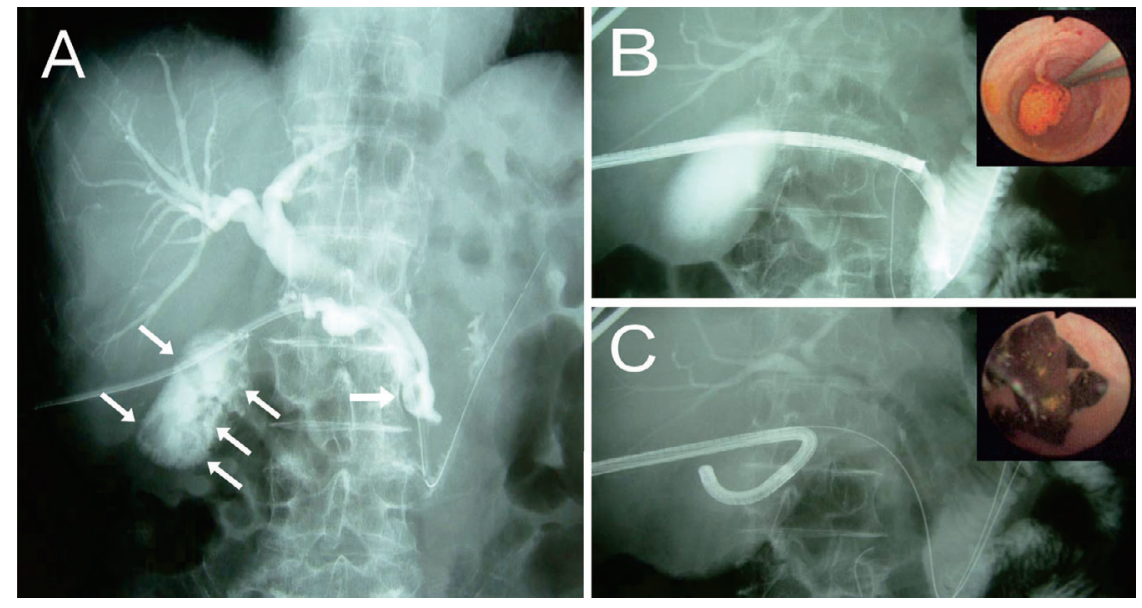

Figure 1. A, Cholangiography representing lithiasis in the common bile duct and gallbladder (arrows). B, Choledochoscope inserted into the common bile duct through the gallbladder and cholecystic duct. Inset, Endoscopic finding of a stone in the common bile duct. C, Choledochoscope removing a number of stones in the gallbladder. Inset, Stones in the gallbladder.

Department of Gastroenterology, Inuyama Chuo Hospital, Aichi

Received for publication March 18, 2007; Accepted for publication June 4, 2007

Correspondence to Dr. Akihiro Mori, a-mori@inuyamachuohospital.or.jp 
CBD (Fig. 1A). After dilatation of the transhepatic tract and cholecystic duct, a choledochoscope was inserted into the CBD through the cholecystic duct (Fig. 1B). Careful and skillful control of a guide-wire was required to pass through the cholecystic duct. However, once the guide-wire reached the duodenum there was no significant difficulty in introducing the catheter and choledochoscope into the cholecystic duct. We then tried to push the CBD stone out of the sphincter with success using the scope (Fig. 1B) because the diameter of the stone was $9 \mathrm{~mm}$ and seemed to be too large to be removed safely via the cholecystic duct using a bascket catheter. Thereafter, we performed PTCS repeatedly and the stones in the gallbladder were removed employing crusher and basket catheters (Fig. 1C), resulting in complete elimination of bile stasis. There was no major complication during the procedure.

Very recently, a guideline for the diagnosis and treatment of acute cholangitis and cholecystitis has been proposed in which early biliary drainage is strongly recommended (5). Biliary drainage can be achieved either by endoscopic transpapillary, percutaneous transhepatic or surgical procedure. Among them early/urgent pre-operative PTGBD has been shown to improve the prognosis of acute cholecystitis particularly in elderly and complicated patients $(2,5)$ as in the present case. Here, we reported simultaneous removal of the lithiasis in the CBD and GB through the PTGBD route. Establishment of dilated cholecystic duct allowed a targeted approach of choledochoscope to a CBD stone as reported previously by the others $(6,7)$. The technique used in the present report may not applicable to all the cases. However, we believe that careful control of a guide-wire would result in the successful insertion into the cholecystic duct in a number of cases, which leads to subsequent PTCS. Thus, we suggest that the present method would be widespread in the treatment of cholelithiasis particularly in patients inapposite for transpapillarly, transhepatic and open approaches.

\section{References}

1. Neuhaus H. Intrahepatic stones: the percutaneous approach. Can J Gastroenterol 13: 464-472, 1999.

2. Tsumura H, Ichikawa T, Hiyama E, et al. An evaluation of laparoscopic cholecystectomy after selective percutaneous transhepatic gallbladder drainage for acute cholecystitis. Gastrointest Endosc 59: 839-844, 2004.

3. Inui K, Nakazawa S, Naito Y, Kimoto E, Yamao K. Nonsurgical treatment of cholecystolithiasis with percutaneous transhepatic cholecystoscopy. Am J Gastroenterol 83: 1124-1127, 1998.

4. Majeed AW, Reed MW, Ross B, Peacock J, Johnson AG. Gallstone removal with a modified cholecystoscope: an alternative to cholecystectomy in the high-risk patient. J Am Coll Surg 184:
273-280, 1997.

5. Miura F, Takada T, Kawarada Y, et al. Flowcharts for the diagnosis and treatment of acute cholangitis and cholecystitis: Tokyo Guidelines. J Hepatobiliary Pancreat Surg 14: 27-34, 2007.

6. Seki S, Sugiyama S, Taniguchi Y, et al. Percutaneous transhepatic transcholecystductual lithotomy for cholecysto- and choledocholithiasis. Tan To Sui 11: 817-824, 1990 (In Japanese without English abstract).

7. Yazawa N, Tobita K, Ohtani Y, et al. Treatment of choledocholithiasis using percutaneous transhepatic transcystic papillary balloon dilatation in an extremely elderly patient. Tando 17: 114-118, 2003 (in Japanese with English abstract).

(C) 2007 The Japanese Society of Internal Medicine http://www.naika.or.jp/imindex.html 